\title{
Homonegativity among first and second generation migrants in Europe: the interplay of time trends, origin, destination and religion
}

ARTICLE in SOCIAL SCIENCE RESEARCH · JANUARY 2014

Impact Factor: 1.27

READS

7

2 AUTHORS:

Koen Van der Bracht

Ghent University

8 PUBLICATIONS 11 CITATIONS

SEE PROFILE

\section{Bart Van de Putte}

Ghent University

54 PUBLICATIONS 178 CITATIONS

SEE PROFILE 


\title{
Homonegativity among first and second generation migrants in
}

\section{Europe: the interplay of time trends, origin, destination and religion}

\author{
Koen VAN der BRacht ${ }^{1}$ \& Bart VAn de Putte \\ Department of Sociology \\ Ghent University
}

\begin{abstract}
Previous studies reported declining disapproval of homosexuality in Europe but have simultaneously identified the decelerating effect of religiosity and the higher disapproval of homosexuality among migrants. In this paper, we address disapproval of homosexuality among first- and second-generation migrants in Europe by assessing (1) period and cohort changes, (2) origin and destination country influences and (3) the role of religiosity. We develop a specific cross-classified multilevel design enabling us to simultaneously examine these influences. We test hypotheses using a subsample of the European Social Survey (ESS), containing 19,878 first and second generation migrants. The analyses lead to three important conclusions. Firstly, disapproval of homosexuality is declining both over time and across cohorts. Secondly, migrants conform to levels of disapproval of homosexuality among natives in the destination country, and this explains the decline among migrants over time. Thirdly, religion has a multi-faceted influence on levels of disapproval of homosexuality among migrants.
\end{abstract}

Keywords: Homonegativity, migrants, HAPC models, religiosity

\footnotetext{
${ }^{1}$ Corresponding author: koen.vanderbracht@ugent.be; Korte Meer 5, 9000 Ghent, Belgium
} 


\section{Introduction}

Concerns in European countries about differences in attitudes between first and second generation migrants on the one hand and non-migrants on the other hand arise in the public media. Especially non-European and often Muslim migrants are considered to have conservative attitudes towards controversial subjects in the personal domain, such as homosexuality. A populist statement in the public debate is the presupposition that Muslims have failed to integrate in the European society because Islamic and Western cultures are irreconcilable (Lucassen, 2005). This difference is also reflected in academic research, with specific attention being devoted to the difference between Muslim migrants and natives (Hooghe and Meeusen 2012; Teney and Subramanian 2010). Scholars have in most cases only studied baseline differences between migrants and non-migrants, thus neglecting the complex interplay of processes influencing migrants and the difference between both. Therefore, the literature is in need of a thorough study of attitudes towards homosexuality among first and second generation migrants in Europe. In this paper we examine disapproval of homosexuality among first and second generation migrants, while focusing on three important aspects: changes over time, origin and destination context effects and religiosity.

A first important aspect which has been overlooked, is possible time trends in disapproval of homosexuality among migrants. During the last decades, a prolonged decline in disapproval of homosexuality has been observed, in Europe and other industrialized nations (Loftus 2001; Treas 2002). The apparent decline in disapproval of homosexuality among natives in Western countries, might, however, not be a simple change in attitudes over time among all individuals, but seems to be largely attributable to cohort replacement (Inglehart 2008; Jaspers, Lubbers and De Graaf 2007; Meeusen and Hooghe 2012; Van de Meerendonk and Scheepers 2004). People growing up in different cohorts, tend to have different opinions due to the socialization context (Inglehart 1989). When it comes to disapproval of homosexuality, older cohorts are, in general, more negative towards homosexuality than younger cohorts (Inglehart 1989). As time goes by, younger cohorts come to age, 
thus changing the composition of the population and hence lowering general levels of disapproval of homosexuality (Loftus 2001). Cohort socialization is often examined as an explanation of levels of homosexuality among natives, but not among migrants. As already indicated, the inertia of attitudes on homosexuality among migrants has not been put to the test: scholars only report higher levels of disapproval among migrants, assuming these levels are static.

Second, the location of socialization can have an important aspect on attitudes as well, apart from the period. There is a large variation in levels of disapproval of homosexuality throughout the world and consequently gay rights (Inglehart 1989). There is considerable variation in Europe as well, with Northern and Western European countries often reporting very low levels and Southern and Eastern European countries reporting somewhat higher levels (Štulhofer and Rimac 2009; van den Akker, van der Ploeg and Scheepers 2013). At the time of writing, an increasing number of countries have legalized, or are in the process of legalizing, same-sex marriages, while other countries maintain and enforce legislation punishing homosexual relations and/or acts with severe sanctions, including the death penalty in some countries. Hence, growing up in a tolerant or non-tolerant country could have an important impact on an individual's stance towards homosexuality. Apart from this contextual effect of the origin country, the destination country can exert an important influence on migrants' attitudes on homosexuality as well. Previous research has demonstrated that migrants often conform to the behavior and attitudes of natives (Maxwell 2010). Therefore, disapproval of homosexuality among migrants needs to be grasped within this complex interplay of origin and destination influences.

A third aspect which has been underdeveloped in the research into disapproval of homosexuality among migrants, is the role of religiosity. Both belonging to a certain denomination and adhering to religious practices, has repeatedly been found as having an increasing effect on disapproval of homosexuality (Hooghe and Meeusen 2012; Jaspers, Lubbers and De Graaf 2007; Loftus 2001; Treas 
2002; van den Akker, van der Ploeg and Scheepers 2013). Given the higher levels of religiosity among migrants in Europe compared to natives, religion is often mentioned as a possible explanation of homophobia (Hooghe and Meeusen 2012). Both individual and contextual religiosity affect moral beliefs about sexuality (Finke and Adamczyk 2008). However, as with disapproval of homosexuality, religiosity itself is influenced by socialization cohorts (Kelley and De Graaf 1997) and, for migrants, with origin and destination country effects (Van der Bracht, Van de Putte and Van de Velde 2014; Van Tubergen 2006). Moreover, religiosity and disapproval of homosexuality are only parts of a wider range of human values which are formed during socialization. Therefore, the effect of religiosity on migrants' disapproval of homosexuality needs to be framed in a broader analysis of the transmission of human values and against the background of socialization within birth cohorts, influenced by aspects of the origin and destination countries.

In this paper, we examine time trends, origin and destination effects and the influence of religiosity. We develop a specific cross-classified multilevel design which enables us to simultaneously model changes over time, as a product of period and cohort changes, as well as assessing influences of origin and destination countries. We apply this specific design to 19,878 first and second generation migrants living in 27 European countries, using four different waves $(2-5 ; 2004-2010)$ of the European Social Survey (ESS).

\section{Theory}

\subsection{Trends over time}

Similar to other attitudes regarding controversial subjects in the personal sphere, such as euthanasia (Jaspers, Lubbers and De Graaf 2007) and sexual intercourse outside marriage (Treas 2002), studies have reported a prolonged decline in disapproval of homosexuality over the last decades, in Europe (Van de Meerendonk and Scheepers 2004), as well as in other Western countries (Inglehart 1989). These changes in attitudes over time are, however, more complex than might seem at first glance. 
Declines might not be general among the whole population, but rather a product of the aging of younger birth cohorts. According to Inglehart (Inglehart 1977), large-scale value changes in Western societies are caused by a shift from an emphasis on material well-being toward an emphasis on the quality of life, due to an improvement in living conditions. As living conditions improve, less time needs to be devoted to satisfying basic needs such as food or economic security, and there is more time to spend on forms of self-expression and individual autonomy (Inglehart 2008). One of the central arguments of this post-materialism theory is that value changes come with generational changes, stating that basic values are formed according to the conditions experienced during the socialization period (Inglehart 2008). Indeed, when it comes to disapproval of homosexuality, or other attitudes on controversial subjects in the personal sphere, the apparent decline over time is actually a product of intergenerational changes (Jaspers, Lubbers and De Graaf 2007; Loftus 2001; Treas 2002; Van de Meerendonk and Scheepers 2004).

In this respect, differences in attitudes on homosexuality among populations over time occur along two different time dimensions: period and cohort trends. First of all, individuals' attitudes can differ relative to the period in which they are surveyed, i.e. period trends. Period trends affect the whole population at a certain moment in time. Second, individuals' attitudes differ due to the period of socialization, i.e. cohort trends. Cohort trends affect all people being born and raised in the same time period. In this paper, we want to examine changing attitudes on homosexuality among first and second generation migrants by looking at the influence of the period and cohort of individuals. To test the post-materialism theory, we apply Hierarchical Age Period Cohort (HAPC) models (Yang and Land 2006) under assumption of certain age effect restrictions (Bell and Jones 2013a).

Over and above these time-effects, migrant generation is also an important aspect of change over time. Although considerable disagreement remains concerning the terms integration and acculturation, a point of agreement is that both integration and acculturation are processes that 
span different generations (Alba \& Nee, 1997; Birman \& Trickett, 2001). Indeed, previous research has demonstrated that migrants conform to attitudes and behaviours of the receiving society in an intergenerational process (Van der Bracht, Van de Putte \& Verhaeghe, 2013; Maxwell, 2010). Therefore, we expect that migrant generation is a fourth form of change over time in levels of disapproval of homosexuality among migrants, whereby second generation migrants will have more positive attitudes on homosexuality.

\subsection{Origin and destination effects}

For migrants, the national origin has an important influence on different aspects of life: health (Huijts and Kraaykamp 2012), religiosity (Van der Bracht, Van de Putte and Van de Velde 2014; Van Tubergen 2006) and partner choice (Beck-Gernsheim 2007) are for instance associated with aspects of the origin country. These differences can be explained by social integration theory, which states that people conform to the norms of the larger group to which they belong, meaning that migrants internalize the attitudes on homosexuality of the origin country (Durkheim 1986). This influence of the origin country can be extended to second generation migrants through socialization within the family (Kelley and De Graaf 1997; Kwak 2003). Given the already mentioned large global variation in attitudes on homosexuality, we expect large differences based on the origin countries of the migrants.

To explain these differences in disapproval among migrants based on origin countries we are somewhat in the dark as only a handful of studies have tried to explain differing national levels, despite the substantial worldwide variation in disapproval of homosexuality. Explanations have mostly been sought in the cultural and religious aspects of countries (Adamczyk and Pitt 2009; Norris and Inglehart 2004). Over and above the influence of individual religiosity, studies have demonstrated that individuals living in contexts with higher levels of religiosity have more conservative attitudes on sexuality in general (Finke and Adamczyk 2008), and homosexuality in 
particular (Adamczyk and Pitt 2009; Finke and Adamczyk 2008; Moore and Vanneman 2003). Among European countries, however, the religious culture of the nation, does not seem to influence disapproval of homosexuality when taking individual religiosity into account (van den Akker, van der Ploeg and Scheepers 2013). When looking at global cross-national variance, individuals living in Muslim nations do have a higher disapproval than those living in Catholic or Hindu nations but do not differ from those in Protestant, Orthodox or Buddhist countries (Adamczyk and Pitt 2009).

These mixed results of the contextual influence of religiosity, however, could be obfuscating different effects, since religiosity is associated with other factors which influence migrant disapproval of homosexuality as well. Following post-materialism theory, it is expected that when levels of human development tend to be higher, people have to devote less time to basic securities (Inglehart 2008). As a consequence, individuals tend to emphasize the importance of human values, such as the quality of life (Inglehart 2008). Individuals growing up with higher feelings of security are more open towards controversial subjects in the personal sphere. Human development is an indication of crossnational variation in the provision of basic securities. Including dummy variables of the dominant religiosity at the national level, however, often groups countries which have similar levels of human development: from a global perspective, European countries are quite similar in the security they offer to their inhabitants, which could explain why there is no effect of contextual religiosity, while levels of insecurity are higher in Middle Eastern and North African countries, which are predominantly Muslim nations. Moreover, these levels of insecurity are linked to levels of religiosity as well, and national human development does succeed in explaining parts of the global variance in religiosity (Immerzeel and Van Tubergen 2013; Norris and Inglehart 2004; Van der Bracht, Van de Putte and Van de Velde 2014). For migrants this means that the impact of the origin country needs to be taken into account and that the impact of the religious culture of the origin country needs to be assessed vis-à-vis the impact of levels of human development. 
Next to the influence of the origin country, social integration takes place in the destination country as well (Durkheim 1986). Migrants conform to the attitudes and behavior of natives in the destination country (Maxwell 2010; Van Tubergen 2006; Van der Bracht, Van de Putte and Verhaeghe 2012). Hence, given the differences in approval of homosexuality in European countries (van den Akker, van der Ploeg and Scheepers 2013), we expect differences in disapproval of homosexuality among migrants according to the levels of disapproval of homosexuality among natives in the destination country. Previous studies have indeed found such contextual influences on migrants' behavior and attitudes: migrants living in more religious contexts tend to be more religious themselves (Van der Bracht, Van de Putte and Van de Velde 2014; Van Tubergen 2006; Van Tubergen and Sindradottir 2011) and second generation migrants have more trust in the parliament in contexts where natives have more trust as well (Maxwell 2010). Given the large variation in disapproval of homosexuality between European countries (Štulhofer and Rimac 2009), we expect differences in levels of disapproval between destination countries and we expect that migrants conform to the levels of disapproval of homosexuality of the natives in the context where they live.

\subsection{The role of religion}

The sociological debate over the influence of religion on attitudes and behaviors is almost as old as the discipline itself. Religiosity is a pervading aspect of virtually all human societies. Moreover, studying the impact of religiosity within the framework of origin and destination country effects is important: as Stark (1996) has pointed out, the influence of religiosity on other aspects of human life is dependent upon the context. The level of religiosity in the context, and the degree of social integration in this context (Durkheim 1986) determine to what extent individuals are influenced by individual levels of religiosity.

The impact of religion on attitudes on sexuality has mainly been explained from two angles: the influence of religious teachings and the influence of religious involvement. When it comes to 
homosexuality, most denominations dogmatize heterosexuality and deem homosexuality as 'unnatural' (Yip 2005). These propositions are spread amongst adherents of denominations through religious teachings and further passed on or enhanced by frequent contact with co-practitioners. In line with this theory, scholars have shown that both being affiliated to religious denominations and practicing more often, enhances more traditional attitudes on homosexuality (Adamczyk and Pitt 2009; Finke and Adamczyk 2008; Hooghe and Meeusen 2012; Jaspers, Lubbers and De Graaf 2007; van den Akker, van der Ploeg and Scheepers 2013). Hence, we expect a positive association between religiosity and disapproval of homosexuality.

This association of religiosity with more traditional opinions is not unique to homosexuality, however. Higher levels of religiosity are associated with more traditional values in general, by inclinations towards avoiding uncertainty and change (Roccas 2005; Schwartz 1992; Schwartz and Huismans 1995). Individuals who are religiously involved give greater priority to conservative values (Meuleman and Billiet 2011) and it can be expected that these traditional values are correlated with disapproval of homosexuality as well. Hence, the influence of religiosity on attitudes on homosexuality need to be assessed vis-à-vis the association of religiosity with traditional values. Therefore, we expect that the association between religiosity and approval of homosexuality will be lower after taking into account traditional values.

\section{Data}

We use data from four different waves $(2-5 ; 2004-2010)$ of the European Social Survey (ESS). The ESS is a two-yearly cross-sectional survey. The central organizing team of the European Social Survey develops the questionnaire, which is then translated to the official languages of the participating countries. Data is then gathered during face-to-face interviews among the resident population aged 15 years or older. Although response rates of at least $70 \%$ are pursued, there is some variation in response rates by countries and waves: from $43.6 \%$ in France in 2004 to $81.4 \%$ in Bulgaria in 2010 . 
Given that the population under study contains all residents of the participating countries regardless of nationality, this dataset is particularly suited for research among first and second generation migrants. Moreover, the dataset contains information which enables us to discern the different relevant contextual levels. A disadvantage of the dataset, however, is that the surveys were conducted using the official languages of the country where they were being taken. This means that less integrated, often meaning less educated, migrants who do not fully master an official language of the country they inhabit are not or less likely to be interviewed. Given that we expect influences of social integration on levels of disapproval of homosexuality, the fact that the dataset contains only the more integrated migrants means we could overestimate the effect of social integration. Therefore, this effect should be considered the effect of native disapproval on relatively wellintegrated first and second generation migrants. The complete dataset of waves two to five contains 194,894 cases. After the selection of migrants and the listwise deletion of missing cases on the variables in the analyses, we retain 19,878 first and second generation migrants.

\section{Methods}

Yang and Land (2006) introduced a so-called Hierarchical Age Period Cohort (HAPC) model, a crossclassified multilevel model which nests respondents in the survey-year on the one hand and the birth cohort on the other hand. Age is then entered at the individual level. They propose it as a way to solve the so-called identification problem, which occurs when trying to simultaneously analyze period, cohort and age. The problem is that age is automatically identified when cohort and period are known. Although the technique has been applied to various outcomes, the suitability for disentangling these different effects has been questioned recently (Bell and Jones 2013b). Models arbitrarily reapportion APC effects, resulting in the interpretation of erroneous results. Moreover, some scholars have gone as far as stating that it is "logically impossible" to disentangle all three effects simultaneously (Glenn 2005: p. 6). 
Bell and Jones (2013a) propose to restrict one of the three time effects in the model to enable the correct estimation of the two other trends. To restrict one effect, however, there need to be firm theoretical reasons to do so. Researchers can restrict effects in two ways, either by removing it from the model or by using strong informative Bayesian priors. The former implies that one has strong theoretical assumptions about the absence of a certain time trend, whereas the latter means a strong theoretical belief in a certain effect size. In both cases, the other time trends are estimated correctly, given that the assumptions regarding the restricted effect are correct. Alternatively, a range of possible effect sizes could be specified and the theoretically most plausible model interpreted. Either of both solutions is ultimately dependent upon theory, the most plausible model can in no way be derived from the results.

To test the post-materialism hypothesis, we want to fully distinguish between period- and cohorteffects. Therefore, we restrict the age effect in our models. Scholars generally agree that age is positively associated with more conservative attitudes in different domains (Truett 1993). Moreover, according to post-materialism theory, cohort-effects are expected. Period-effects on the other hand are expected according to social integration theory (Durkheim 1986) and the apparent decline in disapproval of homosexuality among natives (Van de Meerendonk and Scheepers 2004). In concordance with Bell and Jones (2013a), we specify a strong informative prior, with a standard deviation of 0.001 . With an effect of 0.01 , we specify a moderately strong effect of age: the difference in expected values between the youngest ( 15 years old) and the oldest ( 98 years old) respondent in our analyses is 0.83 , which is relatively high on a 5 -point scale. However, as Bell and Jones (2013a) suggested, we also conducted sensitivity analyses by specifying different strong informative priors, with a range of 0 to 0.025 , corresponding with a difference in expected values of respectively 0 and 2.075 . The results of these additional analyses are discussed in the discussionsection. 
Another problem with the availability of data at the moment is a lack of waves of repeated crosssectional surveys to correctly estimate variance at the period level, hence the period effect. The most frequently applied rule for higher-level units in multilevel modeling is a minimum of 30 (Maas and Hox 2004). When applying Bayesian estimation procedures, however, simulation studies have indicated that a higher-level sample size of even less than 15 units produces reliable estimates, contrary to the estimates produced by maximum likelihood-algorithms (Stegmueller 2013). The more complex the models become, the higher the required number of higher-level units to obtain reliable estimates. When it comes to the cohort-level of HAPC models, this seldom creates problems: even when constructing five-year intervals as birth cohorts, 15 cohorts only cover 75 years, which is a more or less reasonable birth cohort-range for most cross-sectional surveys focusing on an adult population. For the period-effect, however, fifteen repeated waves of cross-sectional surveys are far less common at the moment. Therefore, the application of HAPC models has been hampered by a lack of available data.

At the time of writing, data of only five waves of the ESS are available. At the two-yearly rate, applying HAPC models to the ESS will have to wait until after 2030 to dispose of fifteen different waves, a sufficient number to apply Bayesian estimation procedures. Given the cross-national nature of the ESS, however, there is a possible solution to overcome this estimation problems for the period effect: considering the different waves as clustered within countries. Such national-level time-series cross-sectional data have the advantage that they enable us to simultaneously model cross-sectional effects, which explain differences between countries, and longitudinal effects, which explain differences within countries over time (Fairbrother 2013). A disadvantage, however, is that these models presuppose that social change happens over time within countries: time trends are each time nested within each survey country. Although this might be the case in many cases, for instance effects of industrialization which has had a different timing in many countries, but might not always be true, for instance when studying the influence of global economic or financial crises. This means 
that social change might be occurring as a general European or even global trend. In this case countries should rather be nested within survey-years than the other way round. Given the limited number of available survey-years at the time of writing, reliably estimating the assumption that survey-years are nested in countries or cross-classified to countries by comparing the model fit to that of the alternative model is not warranted. Therefore, we have to assume the nesting of surveyyears within countries. For this paper, however, social integration theory supports this assumption: people conform to the norms of the social group to which they belong. Previous research has indicated that social integration takes place at even more local levels than the national (Van der Bracht, Van de Putte and Verhaeghe 2013). Therefore, we assume that periodic changes in levels of disapproval of homosexuality take place within countries and therefore nest survey-years within survey-countries.

In order to analyze origin and destination country effects, we cross-classify origin and destination with the other levels. This results in the multilevel design depicted in figure 1. Migrants are nested in four different levels, resulting in a cross-classified model containing five different levels. Firstly, migrants are nested within birth cohorts. Thirty birth cohorts with a length of three years have been calculated, with a range of 1908 up until 1997. Secondly, migrants are nested within origin countries. First generation migrants were assigned to their country of birth, second generation migrants where assigned to the country of birth of the foreign-born father, or that of the mother in the case where only the mother is foreign-born. Given that information on the origin was only available at the national level from the second wave of the ESS onwards, we limit our analyses to waves 2-5. This results in a total of 162 different origin countries. Thirdly, migrants are nested within destination countries. The dataset contains all countries participating in waves 2-5 of the ESS, after excluding Israel, Russia and Turkey, resulting in 27 different European countries. Fourthly, migrants are nested within survey-years which are again nested in destination countries, ranging from 2004-2010. Given that not every country has participated in every wave, there are 93 different country-years in the 
dataset. The models were estimated in the statistical software package MLwiN² (Rasbash et al. 2009) by the Markov Chain Monte Carlo (MCMC) estimation procedures (Browne 2012).

\subsection{Dependent variable}

Disapproval of homosexuality is a single-item measure based on the opinion of the respondents with regards to the following statement: "Gay men and lesbians should be free to live their life as they wish". Answers were recorded on a five-point scale ranging from 'Agree strongly' to 'Disagree strongly'. This measure has already been used successfully in previous research (Meeusen and Hooghe 2012; van den Akker, van der Ploeg and Scheepers 2013). In line with previous research, the dependent variable will be analyzed as a metric variable.

\section{FIGURE 1 ABOUT HERE}

\subsection{Independent variables}

Migrant generation is based on the countries of birth of respondents and their parents. First generation migrants are respondents who are born outside the country in which they are surveyed, with both parents being born abroad too. ${ }^{3}$ Migrants are considered second generation if they are born in the country of the survey with at least one parent being born abroad.

Religious affiliation is a categorical variable comprising six categories, based on the answers to the questions whether respondents consider themselves as belonging to any particular religion or denomination and if so, which one. Respondents were given eight possible answers, including the categories Roman Catholic, Protestant, Eastern Orthodox, Other Christian denomination, Jewish, Islamic, Eastern religions and Other non-Christian religions. Both variables have been combined to

\footnotetext{
${ }^{2}$ Version 2.27

${ }^{3}$ Thus excluding natives who are born during temporary stays abroad, or for instance in former colonies of the country in which they were surveyed.
} 
construct one variable with the following six categories: Roman Catholic, Protestant, Eastern Orthodox, Islamic, Other and None.

Subjective religiosity is a metric variable, based on the answer on an eleven-point scale to the question "Regardless of whether you belong to a particular religion, how religious would you say you are?". The scale has a range from 'Not at all religious' (0) to 'Very religious' (10).

Praying is based on the question "Apart from when you are at religious services, how often, if at all, do you pray?". Answers varied in a 7-point scale ranging from 'Every day' (1) to 'Never' (7). Scores were dichotomized by grouping scores 1 to 3 to create the category 'Once a week or more' (1) and 4 to 7 for 'Less than once a week' (0). With this variable we assess the impact of religious observance.

Although scholars have most often assessed the impact of religious service attendance as a measure of religious observance and religious participation, the measure of service attendance has proven to be cross-nationally inequivalent (Meuleman and Billiet 2011). Given that this inequivalence is due to the fact that the indicator does not function for Muslim women, this is an issue which cannot be disregarded for research taking into account effects of migrant religiosity. The other indicators, subjective religiosity and praying are on the contrary indeed cross-culturally equivalent (Meuleman and Billiet 2011).

Conservation is a value dimension: a higher-order dimension of basic types of human values as postulated by Schwartz (Schwartz 1992). The ESS contains a shorter version of the Portrait Values Questionnaire (PVQ): 21 items instead of the 40 items in the PVQ as originally developed by Schwartz. These items are portraits of 21 different people, describing each time that person's goals and aspirations thus pointing to the importance of this value for that person. Respondents were asked to indicate how much they identify with each person, on a six-point Likert scale ranging from 
'very much like me' to 'not like me at all'. The statements have been gender-matched with the respondents. The underlying values of the scales have shown relatively good results in cross-cultural equivalence tests (Davidov 2008). Conservation represents three human values: tradition, conformity and security. All three values are based on two items in the questionnaire, for which the male wording can be found in table $\mathrm{A} 2$ in the appendix. This scale has been calculated by taking the mean scores of each item, with a range of 1 to 6.

\section{TABLE 1 ABOUT HERE}

\subsection{Contextual variables}

To include simultaneously both a longitudinal and a cross-sectional effect of native disapproval at the destination level, the longitudinal effect at the period level is group mean-centered, as described in Fairbrother (2013). In this way, the longitudinal effect of native disapproval is orthogonal to the cross-sectional effect.

Longitudinal native disapproval of homosexuality is a contextual variable which has been aggregated from the dataset. For each year and each destination country we calculated the average disapproval of homosexuality, based on the same question which has been used for the dependent variable, for all natives, i.e. all respondents in the dataset but excluding first and second generation migrants. As already indicated, the mean disapproval of homosexuality, i.e. the cross-sectional native disapproval, is than subtracted from this variable.

Cross-sectional native disapproval of homosexuality is again aggregated from the dataset. For each destination country we calculated the average disapproval of homosexuality. Both native disapproval variables are based on 154,330 natives in the ESS dataset. 
Human Development Index is a composite index indicating the general human development of countries based on the life expectancy at birth, the adult literacy rate and the natural logarithm of gross domestic product per capita at purchasing power parity. We used the HDI of 2002 for all origin countries (United Nations Development Programme 2004). Exceptions here are in the case of 'historical countries' which ceased to exist before 2002 but which were indicated by respondents as origin, e.g. USSR. In that case we used the HDI from the last available year before the dissolution of that country.

National religious culture is a categorical variable indicating the dominant religious tradition in the origin country. A country has a certain religious tradition if $60 \%$ of more of its inhabitants adhere to that religion (Norris and Inglehart 2004). The information on adherents to denominations is derived from the Fractionalization-database (Alesina et al. 2003). When none of the denominations which has adherents in a certain country reaches $60 \%$, that country is considered to have a diverse religious tradition. This results in a variable with seven categories: Catholic, Protestant, Christian (i.e. countries with mixed Protestant and Catholic denominations), Islam, Eastern, Diverse and Other.

\subsection{Control variables}

We control for sex, socio-economic status (SES) and education. Sex is a dichotomous variable with categories 'Male' (0) and 'Female' (1). Socio-economic status is a metric variable. Professions were coded in the dataset following the ISCO-88 classification system. These have been recoded into the International Socio-Economic Index (ISEI) of Ganzeboom, De Graaf and Treiman (1992) The scale has a range from 16 to 90. Respondents with no current employment were given a score of 30, which corresponds with the occupational prestige of people living on an allowance (Treiman 1977). Education is a metric variable measuring the years of full-time education the respondents have completed. 


\section{Results}

Table 1 displays the descriptive statistics of the dependent and independent variables. We notice that the mean of first and second generation migrants' disapproval of homosexuality is 2.31 , which is close to agreeing that gays and lesbians are free to live their life as they wish.

Table 2 displays the results of the cross-classified multilevel analyses of disapproval of homosexuality. We present three different models: (1) a null-model, containing only the intercept, (2) a model containing the appropriate parameters of period, cohort and age and an interaction of generation with the cohort-specification and (3) the final model containing all individual and contextual effects. For each model the table displays the effect coefficients, variance components, corresponding standard errors and DIC fit statistic (Spiegelhalter et al. 2002). From the variance decomposition of the null-model, we notice that there is substantial variance at the higher levels:

$22.3 \%$ (i.e. $\rho=\left(\sigma_{\text {cohort }}+\sigma_{\text {origin }}+\sigma_{\text {destination }}+\sigma_{\text {period }}\right) /\left(\sigma_{\text {cohort }}+\sigma_{\text {origin }}+\sigma_{\text {destination }}+\right.$ $\left.\left.\sigma_{\text {period }}+\sigma_{\text {individual }}\right)\right)$ of first and second generation migrants' levels of disapproval of homosexuality are influenced by the origin country, the destination country, the birth cohort and the period in which they are surveyed. This is relatively high, compared to for instance educational research, where the variance at the higher levels are commonly between $5 \%$ and $20 \%$ (Snijders and Bosker 2011), but in accordance with previously reported levels for analyses among first generation migrants which take into account both the origin and destination (Van der Bracht, Van de Putte and Van de Velde 2014; Van Tubergen 2006). Migrants display the highest correlations according to respectively the origin country (8.0\% variance), birth cohort (7.3\%) and destination country (6.3\%). At the destination-period level, however, the variance is limited, with a correlation of only $0.6 \%$ between individuals being surveyed in the same period and country. The remaining $77.7 \%$ of the variance is due to individual differences between first and second generation migrants. In what follows we first examine changes over time. Next, we turn to the individual effects on disapproval of 
homosexuality. In the last part of the results we focus on the effects at the destination- and originlevel.

\subsection{Trends in homonegativity}

We look at the results of model one to evaluate time-trends in disapproval of homosexuality among first and second generation migrants in Europe. Model one estimates a full HAPC-model by including a linear effect of period, a quadratic specification of birth cohorts and a linear effect of age. Given the informative priors imposed on the age effect, we will only interpret the effects of period and cohort. To enable the interpretation of the cohort-trend for both migrant generations, we depict the cohorttrends in figure 2.

\section{FIGURE 2 ABOUT HERE}

Over the period 2004-2010, we notice a decline in disapproval of homosexuality among first and second generation migrants. This decline is, quite substantial: over the six years under study, there is a predicted decline of about $0.166(-0.035 ; p<0.001)$, on the five-point scale. If this trend would be replicated in the future, the decline would be rather substantial over a longer period. The cohortdifferences are, on the contrary, quite substantial. For both generations we notice a decline up to the cohorts born in the 1960's. For cohorts born during and after the sixties, there is a stagnation in disapproval of homosexuality among both generations. The decline is especially marked among second generation migrants: from levels of disapproval close to 3 among the oldest birth cohorts, we notice levels below 2 for cohorts born in the seventies and eighties, which means that while older second generation birth cohorts in general have a neutral stance towards the statement that gays and lesbians are free to live their life as they which, younger cohorts agree or even strongly agree. At the right end of the graph, however, we notice a slight increase in disapproval among the youngest second generation birth cohorts. Given the interaction with the cohort trends, the generation effect 
differs according to the birth cohort. Looking at figure two, we notice that in the oldest birth cohorts second generation migrants disapprove more of homosexuality than their first generation counterparts, while among cohorts born from the thirties onwards second generation migrants clearly disapprove less. ${ }^{4}$ The gap between both generations is the highest in cohorts born in the seventies and eighties. Hence, we can conclude that disapproval of homosexuality is declining among migrants, in general during the last six years, but additionally across birth cohorts and between generations.

\section{TABLE 2 ABOUT HERE}

\subsection{Individual influences}

In the second model in table 2, all effects are added to the time-specifications. With regards to the control variables, we notice that female migrants, migrants with a higher socio-economic status and migrants with a longer period of education disapprove less of homosexuality. These results are in line with previous research (Loftus 2001).

Regarding the influence of religiosity on disapproval of homosexuality, we notice that religion is associated negatively with tolerance towards homosexuality, even after controlling for traditional values. Compared to Catholic migrants, all migrants adhering another denomination disapprove more often of homosexuality. Especially Muslim migrants $(0.427 ; p<0.001)$ disapprove more often of homosexuality than Catholic migrants, while migrants adhering to the other denominations all exhibit moderate differences compared to Catholic migrants. Non-adhering migrants disapprove significantly less of homosexuality than Catholic migrants. Moreover, as expected, the influence of religiosity on disapproval of homosexuality is not limited to the influence of adhering. A higher

\footnotetext{
${ }^{4}$ Although the higher levels among older second generation birth cohorts could be due to unreliable estimates given small (or 0) group sizes in the oldest cohorts, additional analyses excluding the first four birth cohorts confirm the results in table 2.
} 
subjective religiosity is associated with a higher disapproval of homosexuality. Furthermore, migrants who pray once a week or more often also approve less of homosexuality than migrants who pray less often. Therefore, we can conclude that religiosity exerts a multi-dimensional positive effect on disapproval of homosexuality, over and above the influence of more traditional attitudes.

We notice that our expectations regarding the influence of conservation are corroborated by the results. The effect of conservation, expressing agreement with more traditional values, is positive. This means that migrants with more traditional values have a more negative stance towards gays and lesbians than their less conservation-oriented counterparts. As already indicated, controlling for conservative values does not alter the influence of religiosity on migrant disapproval of homosexuality.

\subsection{Contextual influences}

At the origin-level, we examine the influence of human development and the national religious culture. As expected, migrants coming from countries with a higher human development display lower disapproval of homosexuality. The fact that migrants coming from countries with better living conditions indeed display more positive attitudes is in concordance with Inglehart's post-materialism theory. National religious culture, on the contrary does not have a clear effect on migrant disapproval of homosexuality. Of all religious cultures, only migrants coming from countries with a diverse religious culture display higher levels of disapproval of homosexuality than migrants coming from Catholic countries. After controlling for the human development in a country, national religious cultures only affect migrant disapproval of homosexuality in a limited way, compared to the effects of individual religiosity.

At the period and destination level, we assess the influence of native disapproval of homosexuality on migrant disapproval of homosexuality. At the destination level, the results indicate a positive 
effect of native disapproval on migrant disapproval of homosexuality. Migrants living in countries where attitudes on homosexuality are more negative among natives, also display more negative attitudes. Next to this cross-sectional effect of native disapproval on migrant disapproval, there is also a positive longitudinal effect of native disapproval on migrant disapproval: over time, migrant disapproval of homosexuality is higher at times when native disapproval of homosexuality is higher. Moreover, the positive longitudinal effect of native disapproval on migrant disapproval explains the declining trend in migrant disapproval over time, given that the period-effect is no longer significant after controlling for the longitudinal effect of native disapproval. The decline over time we observed in previous models, seems to be influenced by the effect of native disapproval on migrant disapproval. The declining disapproval of homosexuality among migrants seems to be due to declining levels of disapproval among natives. In this respect, native disapproval of homosexuality is highly associated with differences in disapproval among migrants.

When we compare the remaining variance in the second and final model to the original variance from the null-model, we notice that our second model is quite successful in explaining the higherlevel variance: $86 \%$ of the total higher-level variance has been explained in the second model, with only $4.3 \%$ remaining variance at the higher levels. At the individual level, however, our second model only explains $6.6 \%$ of the original variance in the null-model. Apart from the period-effect, the interpretation of the other time trends is not altered by the inclusion of individual and contextual effects. Hence, we can conclude that our models are quite successful at the higher levels, but better results at the individual level are needed.

Additional sensitivity analyses ${ }^{5}$ indicate that het time trends do not vary according to origin countries of migrants, the denomination of migrants and that the effects of individual denominational affiliation does not vary across origin or destination countries. To grasp the relative contribution of

\footnotetext{
${ }^{5}$ Models not shown but available upon request from the authors.
} 
the different contexts, we look at the variance decomposition in the null-model. We notice that each of them are almost evenly influential, with the origin country exerting slightly more influence on migrants than the other contexts. Regarding the relative importance of time trends, we notice that the baseline trends are almost equal. However, given the long time span, there is a huge difference between younger and older cohorts, as already indicated. Future research would do well to examine the period effect further and map out whether the trend persists.

\section{Conclusion and discussion}

In this paper, we examined disapproval of homosexuality among first and second generation migrants in Europe. We focused on trends over time, origin and destination influences and the role of religion on attitudes on homosexuality. Cross-classified multilevel designs allowing to disentangle both period and cohort influences on the one hand and origin and destination influences on the other hand have been developed. Subsequently, these designs have been applied to data from the European Social Survey (ESS) containing 19,878 individuals, which are nested in 93 destination-years, 27 destination-countries, 162 origin-countries and 30 birth cohorts. From this analyses, we derive three important conclusions.

First of all, we notice a decline in disapproval of homosexuality among migrants living in Europe in a multi-faceted way. During the years under study, from 2004 until 2010, there has been a slight decline in disapproval of homosexuality. The results indicate that this decline can be explained by social integration into the destination country: migrants conform to the declining trend in disapproval among natives. Next to this general decline among migrants, younger birth cohorts disapprove less of homosexuality than older birth cohorts, although this decline has stagnated somewhat from the 1960's onwards. A third and final form of decline in disapproval among migrants is an intergenerational one: second generation migrants have more positive attitudes on homosexuals than their first generation counterparts. The results indicate the dynamic pattern of 
disapproval of homosexuality, which, as already indicated, conforms to the declining trend among natives in Europe. Based on our results, we discern no pattern of diverging trends among natives and migrants, on the contrary.

Second, migrants' attitudes on homosexuality are associated with aspects of both the origin and destination country. Migrants coming from the same origin country have relatively similar attitudes on homosexuality. These attitudes are influenced by levels of human development in the origin country, thus corroborating the post-materialism hypothesis. Migrants from countries with higher levels of human development display more positive attitudes on homosexuals. At the other side of the spectrum, migrants conform to the levels of disapproval in the destination country. Social integration in the destination country seems to be taking place when it comes to attitudes on homosexuality, even when considered longitudinally. When migrants are socially integrated, they learn the attitudes of natives more, through interactions with natives, for instance at work places or by being a member of certain organizations. This finding is in line with other studies who found effects of social integration on, for instance, religiosity among first and second generation migrants (Van Tubergen 2007; Van der Bracht, Van de Putte \& Verhaeghe, 2013). Further research could examine whether the effect of native disapproval is affected by the level of social integration in the host society. With this study, we have also demonstrated that taking into account influences of the origin and destination country when studying disapproval of homosexuality is a valuable approach, in line with previous research (Van Tubergen 2007).

Third, religiosity exerts a strong influence on migrant disapproval of homosexuality. The influence of religion on attitudes on homosexuality is versatile: not only adhering a certain denomination, but also subjective religiosity and the frequency of praying influences the attitudes on homosexuality. This impact of religiosity holds when taking into account how having conservative values affect attitudes on homosexuality. This indicates that individual religiosity indeed exerts a strong influence 
on stances towards homosexuality among migrants, regardless of conservative opinions. Although we only examined the influence of personal dimensions, further research would do well to tackle the role of assessing the influence of service attendance. Given the social nature of service attendance and the effects of social integration in the destination country, service attendance might have a different impact on attitudes towards homosexuality. At the contextual level, on the other hand, there is no clear pattern of national religious cultures on migrant attitudes. Migrants coming from Muslim countries or countries with diverse religious traditions have a higher disapproval than migrants coming from countries with a Roman Catholic tradition, but all other countries do not differ from Roman Catholic countries, after taking into account the levels of human development in those countries. Except from Muslim countries, the religiosity of the country of origin does not affect migrant's disapproval of homosexuality.

The question how social change takes place in societies, as a product of period influences affecting the whole population in a general way, or as a product of cohort influences, affecting individuals in the same birth cohort in a similar way, is central in sociological, demographical and historical research. When it comes to sociological survey-research, however, the full possibilities of studying the timing and occurrence of social change has not been fully exploited. Combining hierarchical ageperiod-cohort models with countries in cross-national repeated cross-sectional surveys offers scholars the ability to do study changes in attitudes and behavior over time among a number of countries. Although the number of waves in the ESS is limited at the time of writing, the availability of additional waves over the next few years should enable scholar to fully estimate the dynamic pattern of individual's attitudes and behavior.

In this paper, however, the research has also suffered from some limitations. First, the results of the period- and cohort-trends are dependent on our assumption regarding the effect of age. However, additional analyses indicate that the results of the period-trend are identical when specifying no age 
effect or a high age effect. ${ }^{6}$ The cohort decline is absent, however, in the case of a high age effect. However, even under the assumption that there is a similar high age effect, the conclusions of the period effect and all other explaining variables hold.

Secondly, some limitations are characteristic to the analyses design. The fact that only four waves of the ESS could be studied means that age can only differ on four time-points when period and cohort are identified. Therefore, we were unable to reliably estimate the effect of age on disapproval of homosexuality among migrants. If more waves of the ESS would have been available, an effect of age could be estimated independently from cohort and period. The effect of age, which was marginally significant in our analyses, could in future analyses with more waves be actually significantly positive, in line with previous research (Loftus 2001). Moreover, as already indicated, we had to assume that the period-changes in attitudes on homosexuality occur within countries, rather than that there is a general period trend with differences between countries being independent from time trends. Our results indicate, however, that there is an effect of time trends within countries, independent from differences between countries. Nonetheless, the growing availability of cross-sectional waves should enable future research to explicitly test this assumption.

Thirdly, although differences due to birth cohorts have been determined, the basic assumption of Inglehart's post-materialism theory, that attitudinal changes occur due to improved living conditions during the socialization period, could not be verified. Given the limited availability of information on living conditions during the socialization period of all birth cohorts under study, we were not able to fully assess the impact of human development during childhood. If such information would be available in future research, the multilevel design which we have developed in this paper can be adapted to include a birth cohorts-within-socialization-location-level, i.e. the origin country for first generation migrants and the destination country for second generation migrants. In this way, we also

\footnotetext{
${ }^{6}$ Respectively an informative prior with means 0 and 0.025 .
} 
enable the possibility of changes within origin countries, similar to the changes within destination countries in the current design.

\section{Acknowledgements}

Funding for this research was provided by the Special Research Fund (BOF) of Ghent University (Grant number B/12322/01). We thank Geert Molenberghs, Rianne Janssen and the anonymous reviewers of Social Science Research for helpful comments on earlier drafts of this article.

\section{References}

Adamczyk, A., Pitt, C., 2009. Shaping attitudes about homosexuality: The role of religion and cultural context. Social Science Research 38(2), 338-351.

Alba, R., Nee, V., 1997. Rethinking assimilation theory for a new era of immigration. International Migration Review 31(4), 826-874.

Alesina, A., Devleeschauwer, A., Easterly, W., Kurlat, S., Wacziarg, R., 2003. Fractionalization. Journal of Economic Growth 8, 155-194.

Beck-Gernsheim, E., 2007. Transnational lives, transnational marriages: a review of the evidence from migrant communities in Europe. Global Networks 7(3), 271-88.

Bell, A., Jones, K., 2013a. Bayesian informative priors with Yang and Land's hierarchical age-periodcohort model. Quality and quantity, DOI: 10.1007/s11135-013-9985-3.

Bell, A., Jones, K., 2013b. Another 'futile quest'? A simulation study of Yang and Land's Hierarchical Age-Period-Cohort model. Demographic Research 30(11), 333-360.

Birman, D., Trickett, E.J., 2001. Cultural Transitions in First-Generation Immigrants Acculturation of Soviet Jewish Refugee Adolescents and Parents. Journal of Cross-Cultural Psychology 32(4), 456-77.

Browne, W.J., 2012. MCMC estimation in MLwiN. Centre for Multilevel Modelling, Bristol. 
Clarke, P. J., 2008. When can group level clustering be ignored? Multilevel models versus single-level models with sparse data. Journal of Epidemiology and Community Health 62(8), 752-758.

Davidov, E., 2008. A cross-country and cross-time comparison of the human values measurements with the second round of the European Social Survey. Survey Research Methods 2(1), 33-46.

Durkheim, E., 1986. Le suicide: etude de sociologie. PUF, Paris.

Fairbrother, M., 2013. Two Multilevel Modeling Techniques for Analyzing Comparative Longitudinal Survey Datasets. Political science research and methods 2(1), 119-140.

Finke, R., Adamczyk, A., 2008. Cross-national moral beliefs: The Influence of National Religious Context. The Sociological Quarterly 49(4), 617-652.

Ganzeboom, H.B.G., De Graaf, P.M., Treiman, D.J., 1992. A standard international socio-economic index of occupational status. Social Science Research 21(1), 1-56.

Hooghe, M., Meeusen, C., 2012. Homophobia and the Transition to Adulthood: A Three Year Panel Study among Belgian Late Adolescents and Young Adults, 2008-2011. Journal of youth and adolescence 41(9), 1197-1207.

Hox, J. J., 2010. Multilevel analysis: Techniques and applications. Routledge, New York.

Huijts, T., Kraaykamp, G., 2012. Immigrants' Health in Europe: A Cross-Classified Multilevel Approach to Examine Origin Country, Destination Country, and Community Effects. International Migration Review 46(1), 101-137.

Immerzeel, T., Van Tubergen, F., 2013. Religion as Reassurance? Testing the Insecurity Theory in 26 European Countries. European Sociological Review 29(2), 359-372.

Inglehart, R.F., 1977. The Silent Revolution: Changing Values and Political Styles Among Western Publics. Princeton University Press, Princeton.

Inglehart, R.F., 1989. Culture shift in advanced industrial society. Princeton University Press, Princeton.

Inglehart, R.F., 2008. Changing values among western publics from 1970 to 2006 . West European Politics 31(1-2), 130-146. 
Jaspers, E., Lubbers, M., De Graaf, N.D., 2007. 'Horrors of Holland': Explaining Attitude Change Towards Euthanasia and Homosexuals in The Netherlands, 1970-1998. International Journal of Public Opinion Research 19(4), 451-473.

Kelley, J., De Graaf, N.D., 1997. National Context, Parental Socialization, and Religious Belief: Results from 15 Nations. American Sociological Review 62(4), 639-659.

Kwak, K., 2003. Adolescents and their parents: a review of intergenerational family relations for immigrant and non-immigrant families. Human Development 46, 115-136.

Loftus, J., 2001. America's liberalization in attitudes toward homosexuality, 1973 to 1998. American Sociological Review 66(5), 762-782.

Lucassen, L., 2005. The immigrant threat: the integration of old and new migrants in Western Europe since 1850. University of Illinois press, Urbana.

Maxwell, R., 2010. Evaluating Migrant Integration: Political Attitudes Across Generations in Europe. International Migration Review 44(1), 25-52.

Meeusen, C., Hooghe, M., 2012. Period, cohort or generational replacement? Explaining the decline in disapproval of homosexuality in Belgium 2002-2010. Paper presented at the International Conference on the European Social Survey (ESS), 23-25 November 2012.

Meuleman, B., Billiet, J., 2011. Religious involvement: its relation to values and social attitudes. In: Davidov, E., Schmidt, P., Billiet, J. (Eds.), Cross-cultural analysis: methods and applications. Taylor and Francis Group, New York, pp. 173-206.

Maas, C.J.M., Hox, J.J., 2005. Robustness issues in multilevel regression analysis. Statistica Neerlandica 58(2), 127-137.

Moore, L.M., Vanneman, R., 2003. Context Matters: Effects of the Proportion of Fundamentalists on Gender Attitudes. Social Forces 82(1), 115-139.

Norris, P., Inglehart, R.F., 2004. Sacred and secular. Cambridge University Press, New York.

Rasbash, J., Charlton, C., Browne, W.J., Healy, M., Cameron, B., 2009. MLwiN Version 2.1. Centre for Multilevel Modelling, Birstol. 
Roccas, S., 2005. Religion and value systems. Journal of Social Issues 61(4), 747-759.

Schwartz, S.H., 1992. Universals in the content and structure of values: Theoretical advances and empirical tests in 20 countries. Advances in experimental social psychology 25(1), 1-65.

Schwartz, S.H., Huismans, S., 1995. Value priorities and religiosity in four western religions. Social Psychology Quarterly 58(2), 88-107.

Snijders, T.A.B., Bosker, R.J., 2011. Multilevel analysis: An introduction to basic and advanced multilevel modeling. Sage Publications Limited, London.

Spiegelhalter, D.J., Best, N.G., Carlin, B.P., van der Linde, A., 2002. Bayesian measures of model complexity and fit. Journal of the Royal Statistical Society. Series B (Statistical Methodology) 64(4), 583-639.

Stegmueller, D., 2013. How Many Countries for Multilevel Modeling? A Comparison of Frequentist and Bayesian Approaches. American Journal of Political Science 57(3), 748-761.

Štulhofer, A., Rimac, I., 2009. Determinants of homonegativity in Europe. Journal of sex research 46(1), 24-32.

Teney, C., Subramanian, S.V., 2010. Attitudes toward homosexuals among youth in multiethnic Brussels. Cross-Cultural Research 44(2), 151-173.

Treas, J., 2002. How cohorts, education, and ideology shaped a new sexual revolution on American attitudes toward nonmarital sex, 1972-1998. Sociological Perspectives 45(3), 267-283.

Treiman, D.J., 1977. Occupational prestige in comparative perspective. Academic Press: New York.

Truett, K.R., 1993. Age differences in conservatism. Personality and individual differences 14(3), 40511.

United Nations Development Programme 2004. Human Development Report. United Nations: New York.

Van de Meerendonk, B., Scheepers, P., 2004. Denial of equal civil rights for lesbians and gay men in the Netherlands, 1980-1993. Journal of Homosexuality 47(2), 63-80. 
van den Akker, H., van der Ploeg, R., Scheepers, P., 2013. Disapproval of homosexuality: Comparative research on individual and national determinants of disapproval of homosexuality in 20 European countries. International Journal of Public Opinion Research 25(1), 64-86.

Van der Bracht, K., Van de Putte, B., Van de Velde, S. 2014. The worst of both worlds? Origin and destination effects on migrant religiosity. Ethnic and Racial Studies DOI:10.1080/01419870.2012.748211.

Van der Bracht, K., Van de Putte, B., Verhaeghe, P.-P., 2013. God bless our children?: the role of generation, discrimination and religious context for migrants' religion in Europe. International migration 51(3), 23-37.

Van Tubergen, F., 2006. Religious affiliation and attendance among immigrants in eight western countries: Individual and contextual effects. Journal for the Scientific Study of Religion 45(1), $1-22$.

Van Tubergen, F., Sindradottir, J.I. 2011. The Religiosity of Immigrants in Europe: A Cross-National Study. Journal for the Scientific Study of Religion 50(2), 272-288.

Yang, Y., Land, K.C., 2006. A mixed models approach to the age-period-cohort analysis of repeated cross-section surveys, with an application to data on trends in verbal test scores. Sociological methodology 36(1), 75-97.

Yip, A.K.T., 2005. Queering religious texts: An exploration of British non-heterosexual Christians' and Muslims' strategy of constructing sexuality-affirming hermeneutics. Sociology 39(1), 47-65. 